\title{
SISTEMA DE PRÉ-AVISO PARA O CONTROLE DA SIGATOKA-AMARELA DA BANANEIRA NO RECÔNCAVO BAIANO ${ }^{1}$
}

\author{
DANÚZIA MARIA VIEIRA FERREIRA², ZILTON JOSÉ MACIEL CORDEIRO ${ }^{3}$, ARISTOTELES PIRES DE MATOS $^{4}$
}

\begin{abstract}
RESUMO - Entre os problemas que afetam a bananicultura brasileira, a Sigatoka-amarela (Mycosphaerella musicola Leach) destaca-se como um dos mais graves, podendo causar perdas superiores a $50 \%$ na produção. O controle químico continua sendo uma das principais alternativas disponíveis. Por isto a utilização de um sistema de monitoramento que possa indicar o momento correto da aplicação dos fungicidas é uma alternativa importante para racionalizar seu uso. O objetivo deste trabalho foi definir o valor de soma bruta no sistema de pré-aviso biológico, que permita reduzir o número de aplicações anuais de defensivos capaz de controlar eficientemente a Sigatoka-amarela na região do Recôncavo Baiano. O ensaio foi conduzido na área experimental do Centro Nacional de Pesquisa de Mandioca e Fruticultura Tropical - Embrapa Mandioca e Fruticultura, da Empresa Brasileira de Pesquisa Agropecuária - EMBRAPA, no município de Cruz das Almas, BA. Testaram-se oito tratamentos, seis utilizando a soma bruta ( 1000; 1300; 1600; 1900; 2200 e 2500), o controle sistemático da doença a cada 21 dias e a testemunha controle. Esses tratamentos foram distribuídos em oito quadras de 48 plantas da cultivar Grande Naine, avaliando-se semanalmente dez plantas por tratamento, quanto à taxa de emissão foliar e incidência da doença nas folhas 2, 3 e 4, indicando a intensidade do estádio mais avançado da lesão presente nas mesmas. Os dados semanais foram usados para o cálculo das respectivas somas brutas, recomendando ou não a aplicação do fungicida (propiconazole $3 \mathrm{~mL}$ mais $1 \mathrm{~L}$ de óleo mineral) . Na colheita coletaram-se os dados de produção e severidade da doença. Apenas os tratamentos controle sistemático, soma bruta 1300 e soma bruta 1600 apresentaram produções estatisticamente diferentes da testemunha sem controle. Considerando a produtividade obtida e o número de aplicações de fungicidas, requeridas durante o ciclo, foi concluído que, para as condições do Recôncavo Baiano, a aplicação do sistema de pré-aviso biológico para o controle químico da Sigatoka-amarela, deve utilizar o valor de Soma Bruta 1600 como indicador da época correta de realização das pulverizações. Nesta condição, houve uma redução de treze para oito aplicações anuais, ou seja, $40 \%$ menos defensivos aplicados, sem perda na produtividade.
\end{abstract} Termos para indexação: banana, doença, Pseudocercospora musae, Mycosphaerella musicola.

\section{FORECASTING FOR THE CONTROL OF BANANA 'YELLOW SIGATOKA' IN THE RECÔNCAVO BAIANO REGION, BRAZIL}

ABSTRACT - Yellow Sigatoka (Mycosphaerella musicola Leach) is one of the most serious banana diseases in Brazil, it can cause yield losses higher than $50 \%$. The main control method has been based on spraying fungicides. The use of a monitoring system able to indicate the appropriate moment for spraying fungicide, is a very important alternative to rationalize its uses. This work aimed to defining a Total Sum value for the biological forecasting system that allows to reduce the number of annual fungicide applications needed for Yellow Sigatoka control in Recôncavo Baiano Region. The work was carried out at Embrapa Cassava \& Fruits, of the Brazilian Agricultural Research Corporation - EMBRAPA, located in the Municipality of Cruz das Almas, State of Bahia. Eight treatments were evaluated: six of them using pre-established Total Sum values of the biological forecasting system $(1000 ; 1300 ; 1600 ; 1900 ; 2200$ and 2500), the sistematic disease control by fungicide spraying every 21 days, and the control treatment without spraying. The treatments consisted of eight plots containing 48 plants each, of the cultivar Grand Naine. Every week, ten plants of each treatment were evaluated for rate of leaf emission and disease incidence in the leaves 2, 3 and 4, in order to determine the highest degree of lesion intensity. The weekly data were used to calculate the respective Total Sum values thus indicating the necessity or not of fungicide application (propiconazole, $3 \mathrm{~mL}$, plus mineral oil, $1 \mathrm{~L}$ ). At harvest, data on production and disease severity were taken. Only three of the evaluated treatments were statistically different from the control treatment without sprayings: fungicide application every 21 days; fungicide application when the Total Sum reached 1,300; and fungicide application when the Total Sum reached 1,600. Considering the yield obtained and the number of fungicide applications required during the crop cycle, it is concluded that, for the Recôncavo Baiano Region, the application of the biological forecasting system for chemical control of Yellow Sigatoka should be performed when the Total Sum reaches 1,600. This system allowed a decrease in the number of fungicide application from thirteen to eight per year, that means $40 \%$ less fungicide used without decreasing yield.

Index terms: banana, disease, Pseudocercospora musae, Mycosphaerella musicola.

\section{INTRODUÇ̃̃O}

A bananicultura é uma atividade de elevada importância econômica e social, respondendo pela produção de alimento básico para as populações carentes de diversos países, mas também presente na mesa de todas as camadas sociais da população. A banana ocupa o segundo lugar em volume de frutas produzidas no Brasil, que é o terceiro maior produtor do mundo, com uma produção de 5.499.970 toneladas, numa área cultivada de 521.285 ha, sendo superado apenas pela Índia e Equador (FAO, 2001).

Um dos grandes problemas da bananicultura nacional é a Sigatoka-amarela, uma doença endêmica, com picos durante o período chuvoso no Brasil. É causada pelo fungo Mycosphaerella musicola Leach, que, ao infectar as folhas, provoca a morte prematura das mesmas, causando perdas superiores a 50\% na produção (Martinez, 1970). $\mathrm{O}$ cultivo de variedades suscetíveis à Sigatoka-amarela torna a aplica- ção de fungicidas uma prática indispensável no controle da doença. Assim, o uso sistemático de produtos químicos, além de aumentar o custo de produção, constitui prática agressiva ao meio ambiente e ao ser humano, podendo ainda selecionar formas resistentes do patógeno. Sistemas de pré-aviso biológico começaram a ser trabalhados por Ganry \& Meyer (1972) e por Ternesien (1985); Fouré (1988) e Marín \& Romero (1992), que procuraram simplificá-los e, consequentemente torná-los práticos. O objetivo do presente trabalho foi estabelecer parâmetros, num sistema de pré-aviso biológico, que permitam um controle químico racional da Sigatoka-amarela no Recôncavo Baiano, estabelecendo quando e com que freqüência as pulverizações deverão ser realizadas. $\mathrm{O}$ método de previsão se baseia na interação entre o estádio de desenvolvimento da folha vela e a severidade da doença nas folhas mais novas, tornando-se uma alternativa altamente importante na racionalização do uso de fungicidas.

\footnotetext{
1(Trabalho 137/2002). Recebido: 30/08/2002. Aceito para publicação: 11/09/2003.

${ }^{2}$ Eng. Agr., M. Sc., ADAB - Barreiras; E-mail: danuzia@cdlmma.com.br

${ }^{3}$ Eng. Agr., D. Sc., Embrapa/CNPMF; E-mail: zilton@cnpmf.embrapa.br

${ }^{4}$ Eng. Agr., Ph. D., Embrapa/CNPMF; E-mail: apmatos@cnpmf.embrapa.br
} 


\section{MATERIALEMÉTODOS}

O sistema de plantio utilizado foi em fileiras duplas, no espaçamento $4 \mathrm{~m} \times 2 \mathrm{~m} \times 2 \mathrm{~m}$; a cultivar foi a Grande Naine (grupo AAA), suscetível à Sigatoka-amarela. $\mathrm{O}$ experimento foi constituído por oito tratamentos, cada tratamento com quarenta e oito plantas, avaliando-se dez plantas de cada tratamento. Os tratamentos utilizados foram: 1. Controle sistemático, normalmente utilizado nos plantios comerciais com aplicação de fungicida a cada 21 dias; 2 . Soma bruta $1000 ; 3$. Soma bruta 1300; 4. Soma bruta 1600; 5. Soma bruta 1900; 6. Soma bruta 2200; 7. Soma bruta 2500 e 8 . Sem controle da doença. Nos tratamentos 2 a 7, o controle foi realizado sempre que o valor da soma bruta era atingido.

A coleta de dados semanais foi feita em dez plantas por tratamento em função do seu desenvolvimento regular e homogêneo. Em uma ficha foram anotados o estádio da folha vela (Brun, 1963) e a incidência (estádio da lesão) da doença nas folhas 2, 3 e 4 (Fouré, 1994). Em outra ficha, foram anotados os dados sobre severidade da doença de acordo com Gauhl (1994). Na época da colheita foram anotados os dados referentes a peso do cacho e severidade da doença.

Os dados coletados nas folhas 2, 3 e 4 incluem o estádio da lesão e a densidade de ataque observada, tomando-se como referência o número de 50 lesões no nível mais elevado de desenvolvimento. $\mathrm{Ou}$ seja, quando se tinha mais de cinquenta lesões do estádio mais desenvolvido, assinalava-se com $(+)$, quando se tinha menos de cinquenta lesões do estádio mais desenvolvido, assinalava-se com (-).

Para o cálculo do valor de soma bruta, os dados referentes ao estádio da lesão e a densidade de ataque nas folhas 2,3 e 4 foram multiplicados pelos coeficientes estabelecidos por Ganry \& Laville (1983).

Com os dados de doença e crescimento do hospedeiro alimentou-se um programa de computador, cedido pela Corporación Bananera Nacional (CORBANA), Costa Rica, que calculou o ritmo de emissão foliar, a soma bruta e o estado de evolução da doença, fornecendo desta forma os parâmetros fundamentais para o desenvolvimento do sistema de pré-aviso. Sempre que o estado de evolução da Sigatokaamarela atingiu os valores de soma bruta preestabelecidos para cada tratamento foi implementado o controle da doença. As aplicações foram feitas utilizando-se atomizador costal motorizado modelo MK 30, com capacidade 20L. O produto utilizado foi o propicanazole, na dosagem de 100 gramas por hectare, em mistura com 5 litros de óleo mineral e água para completar aproximadamente 22 a 23 litros da calda por hectare. $\mathrm{O}$ delineamento estatístico utilizado foi o inteiramente casualizado, com oito tratamentos e 10 repetições.

Os dados de produção foram submetidos à análise de variância, utilizando-se o programa estatístico SAEG O teste de Tukey, a 5\% de probabilidade, foi usado para comparação das médias.

\section{RESULTADOS E DISCUSSÃO}

As curvas de evolução da doença (Figura 1) mostram um efeito sazonal bastante significativo, com elevada severidade nos meses de maio a agosto, decrescendo a partir daí e atingindo níveis pouco expressivos no período compreendido entre os meses de dezembro a março, que corresponde ao período menos chuvoso (Stover, 1968; Pérez, 1978). Isto mostra o quanto os fatores umidade e temperatura são importantes na epidemiologia dessa doença.

Analisando separadamente os tratamentos, observa-se que as curvas seguem a mesma tendência com pequenas variações nos diferentes meses. Os tratamentos 1, 2, 3 e 4 apresentam os menores valores de soma bruta. O tratamento que mais se aproxima deste grupo é o 5 , todavia, observa-se que os valores da soma bruta estão sempre mais altos. Como os tratamentos, do um ao oito, decrescem em rigor em relação ao controle da Sigatoka-amarela, pode-se dizer que os resultados estão refletindo o aumento da severidade da doença com a redução do rigor empregado no controle químico.

Comparando a curva apresentada pelo tratamento um (controle sistemático) em relação às demais (Figura 1), observa-se que a mesma apresenta variações menos bruscas em relação aos outros tratamentos. Isto pode ser creditado à manutenção da doença em níveis sempre baixos com a utilização de fungicida em intervalos pré-fixados. Já os demais tratamentos apresentam curvas com variações mais bruscas, representando as intervenções com fungicida, feitas quando a severidade da doença atingia o estádio de evolução pré-estabelecido. Ainda comparando o tratamento um com os demais, observa-se que, em alguns pontos, os tratamentos dois (SB 1000), três (SB 1300) e até mesmo o quatro (SB 1600) apresentaram valores de soma bruta menores do que o mesmo. Isto mostra que as aplicações de fungicidas, quando realizadas no momento correto, podem apresentar efeito benéfico até mais acentuado do que aquelas aplicações realizadas em intervalos regulares, sem considerar o nível de severidade da doença.
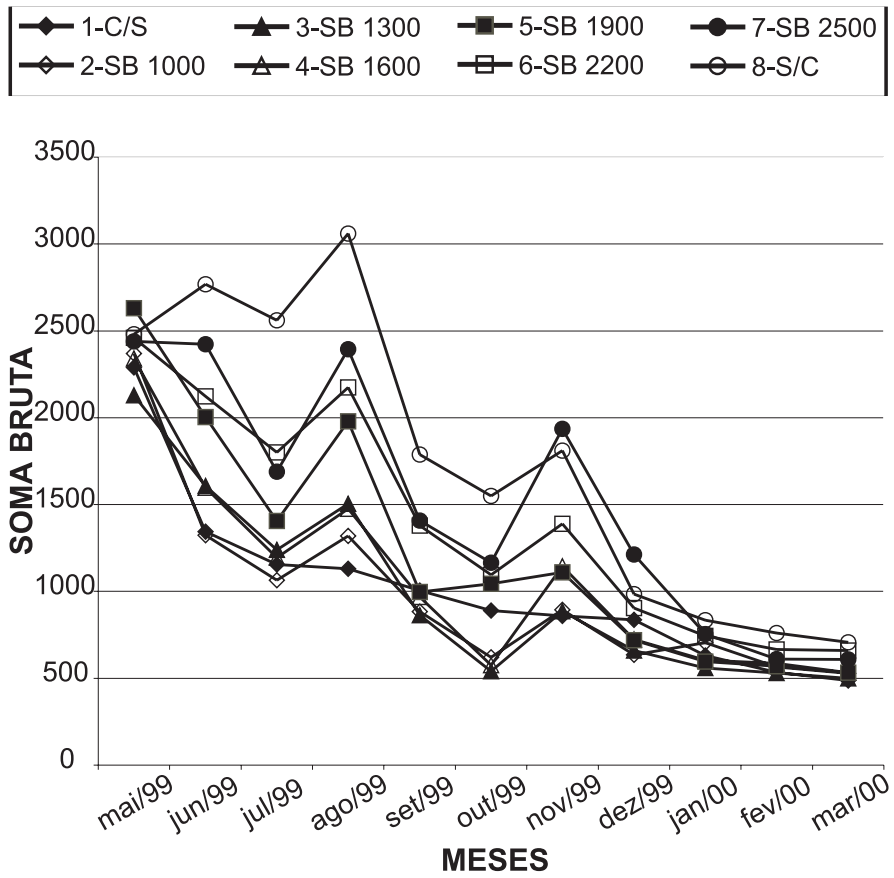

FIGURA 1 -Evolução da Sigatoka-amarela, representada pela média mensal da soma bruta, obtida semanalmente, mediante leitura do estádio de lesão da doença, em plantas da cultivar Grande Naine, entre maio/99 e março/00, onde: 1 - Controle sistemático (C/S) ; 2 - Soma Bruta (SB) 1000; 3 - Soma Bruta (SB) 1300; 4 - Soma Bruta (SB) 1600; 5 - Soma Bruta (SB) 1900; 6 - Soma Bruta (SB) 2200; 7 - Soma Bruta (SB) 2500; 8 - Sem controle (S/C). Embrapa Mandioca e Fruticultu$\mathrm{ra}$, Cruz das Almas, Bahia, 2000.

Analisando a curva de progresso da doença, obtida com os dados de soma bruta (Figura 1), os dados de produção e o número de atomizações efetuados por tratamento (Tabela 1), conclui-se que entre os tratamentos que apresentaram produções significativamente superiores à testemunha sem controle (tratamento oito), o tratamento quatro é o que mais se destaca, uma vez que possibilitou a redução do número de pulverizações de treze (controle sistemático) para oito e sem comprometer a produção, a qual foi similar àquela apresentada pela testemunha absoluta (controle sistemático). Estes resultados têm importantes implicações financeiras porque reduzem o custo de produção ao reduzir o número de aplicações em $40 \%$, é preservacionista ao reduzir a quantidade de pesticidas jogados no ambiente e, além disto, reduz a pressão de seleção sobre o patógeno e o aparecimento de variantes resistentes aos fungicidas que, conforme enfatizado por Boreau et al. (1982) e Bureau (1984), é hoje um dos grandes problemas vividos pelas regiões de cultivo intensivo de banana.

No tocante à produção, os tratamentos um (controle sistemático), três (SB 1300) e quatro (SB 1600) foram os que possibilitaram os maiores pesos médios de cacho com $31,18 \mathrm{~kg}, 30,69 \mathrm{~kg}$ e $30,86 \mathrm{~kg}$, respec- 
TABELA 1 - Peso médio dos cachos e número total de aplicações por tratamento, da mistura propiconazole + óleo mineral, para controle da Sigatokaamarela, em bananeiras 'Grande Naine'. Embrapa Mandioca e Fruticultura, Cruz das Almas, Bahia, 2000.

\begin{tabular}{|c|c|c|c|c|c|c|c|c|}
\hline Tratamentos $^{\mathrm{z}}$ & 1 & 2 & 3 & 4 & 5 & 6 & 7 & 8 \\
\hline $\mathrm{N}^{\circ}$ total de aplicacões & 13 & 12 & 11 & 8 & 10 & 7 & 4 & 0 \\
\hline Peso médio dos cachos & $31,1 \mathrm{a}$ & $27,9 \mathrm{ab}$ & $30,7 a$ & $30,8 \mathrm{a}$ & $24,5 \mathrm{ab}$ & $26,1 \mathrm{ab}$ & $23,9 \mathrm{ab}$ & $22,1 b$ \\
\hline
\end{tabular}

z 1 - Controle sistemático (pulverização a intervalo de 3 em 3 semanas); 2 - Soma bruta 1000; 3 - Soma bruta 1300; 4 - Soma bruta 1600; 5 - Soma bruta 1900; 6 Soma bruta 2200; 7 - Soma bruta 2500; 8 - Sem controle. Médias seguidas pelas mesmas letras não diferem estatisticamente entre si, pelo teste de Tukey ao nível de $5 \%$ de probabilidade.

tivamente, diferindo significativamente da testemunha não pulverizada (Tabela 1). Ainda com relação à produção, os tratamentos dois (SB 1000), cinco (SB 1900), seis (SB 2200) e sete (SB 2500), embora sendo estatisticamente semelhantes ao um, três e quatro, não diferiram significativamente do tratamento testemunha sem controle, indicando que os níveis de redução da doença obtidos com esses tratamentos não foram suficientes para evitar quedas na produção.

Ressalta-se que o tratamento dois possibilitou elevada eficiência de controle da Sigatoka-amarela, expressa pelos valores médios reduzidos para soma bruta (Figura 1). Assim sendo, o menor peso médio do cacho evidenciado nesse tratamento (Tabela 1) deve ser atribuído a outros fatores relacionados com o processo produtivo e não a perdas causadas pela doença. Vale salientar ainda que a fertilidade do solo, além de propiciar maiores produções, também interfere na severidade da Sigatoka-amarela sobre a bananeira (Cordeiro \& Matos, 2000). Por estas razões e considerando a produção obtida (Tabela 1), pode-se dizer que, na Região do Recôncavo Baiano, o controle químico da Sigatoka-amarela deve ser iniciado quando a Soma Bruta atingir o valor 1600.

\section{CONCLUSÕES}

Para as condições do Recôncavo Baiano, a aplicação do sistema de pré-aviso biológico, para o controle da Sigatoka-amarela, deve utilizar o valor de soma bruta 1600 como indicador para se efetuar o controle químico da doença. Todavia, é importante estar atento para a curva de progresso da doença e fatores climatológicos, principalmente previsão de chuva.

\section{REFERÊNCIAS BIBLIOGRÁFICAS}

BRUN, J. La Cercosporiose du bananier in Guinée: etude de la phase Ascorporée du Mycosphaerella musicola Leach. 1963. 1967. Tese (Doctor em Science) - IFAC, Paris, 1963 (Série A. Orsay, 35).

BUREAU, E. L'avertissement climatique dans le cadre d'une stratégie nouvelle de traitments contre la cercosporiose du bananier em Guadalupe. Fruits, Paris, v. 39, n. 7/8, p. 441-447, 1984.

BUREAU, E.; GANRY, J.; ZAPATER, M.F.; LAVILLE, E. Les cercosporioses du bananier et leurs traitements. Evolution des populations pathogènes. Fruits, Paris, v.37, n.11, p.665-671, 1982.

CORDEIRO, Z. J. M.; MATOS, A. P. de. Doenças fúngicas e bacterianas. In: CORDEIRO, Z. J. M. (Coord.). Banana: fitossanidade. Brasília: Embrapa Comunicação para Transferência de Tecnologia, 2000. p.
36-65. (Frutas do Brasil, 8).

FAO. Produção de banana. Disponível em: $<$ http://apps.fao.org $>$. Acesso em: 27 nov. 2001

FOURÉ, E. Leaf spot disease of banana and plantain caused by Mycosphaerella musicola and $M$. fijiensis. In: INTERNATIONAL NETWORK FOR THE IMPROVEMENTOF BANANAAND PLANTAIN, 1994, Honduras. The improvement and testing of Musa : a global partnership. Montpellier: INIBAP, 1994. p.47-53.

FOURÉ, E. Stratégies de lutte contre la cercosporiose noire des bananiers et des plantains provoquée par Mycosphaerella fijiensis Morelet. L'avertissement biologique au Cameroun. Evaluation des possibilités d'amélioration. Fruits, Paris, v. 43, n. 5, p. 269-274, mai, 1988.

GANRY, J.; LAVILLE, E. Les cercosporioses du bananier et leurs traitment; evolutions des méthodes de traitment. I. Traitments fongicides 2. Avertissement. Fruits, Paris, v. 38, n. 1, p. 3-20, jan. 1983.

GANRY, J.; MEYER, J. P. La lutte controlée contre le cercospora aux Antilles. Bases climatiques de l'avertissement. Tecnique d'observation et numération de la maladie. Fruits, Paris, v.27, n. 11, p. 767-774, nov. 1972.

GAUHL, F. Epidemiology and ecology of black Sigatoka (Mycosphaerella fijiensis Morolet) on plantain and banana (Musaspp) in Costa Rica, Central América. Montpellier: INIBAP, 1994. 120p.

IBGE. Levantamento sistemático da produção agrícola. Disponível em:<http://www.sidra.ibge.gov.br>. Acesso em: 09 fev. 2001.

MARÍN VARGAS, D.; ROMERO CALDERÓN, R. El combate de la Sigatoka negra. San José: CORBANA. Departamento de Investigaciones, 1992.21p. (Boletin, 4).

MARTINEZ, J.A. O mal-de-Sigatoka e sua importância econômica para a bananicultura do estado de São Paulo. O Biológico, São Paulo, v.36, p. 271-280, 1970.

PÉREZ, L. Control de Mycosphaerella musicola (Sigatoka) com aceite y mezclas de fungicidas sistémicos en aceite mineral. Agrotecnia de Cuba, Cuba, v.10, n. 1, p. 95-104, 1978.

STOVER, R. H. Leaf spot of banana caused by Mycosphaerella musicola. Perithecia and sporodochia production in different climates. Tropical Agriculture Journal, Trinidad, v. 45, p. 1-12, 1968.

STOVER, R. H. A proposed international scale for estimating intensity of banana leaf spot (Mycosphaerella musicola). Tropical Agriculture , Trinidad, v.48, n.3,p. 185-196, july 1971.

TERNISIEN, E. Les cercosporioses des bananiers et plantains: méthodes de lutte: avertissements: perspectives au Cameroun. Paris: ENITH, 1985. (Mémoire de fin d'études). 